\title{
Extension of Eaves Theorem for Determining the Boundedness of Convex Quadratic Programming Problems
}

\author{
Huu-Quang Nguyen*, Van-Bong Nguyen and Ruey-Lin Sheu
}

\begin{abstract}
It is known that the boundedness of a convex quadratic function over a convex quadratic constraint (c-QP) can be determined by algorithms. In 1985, Terlaky transformed the said boundedness problem into an $l_{p}$ programming problem and then apply linear programming, while Caron and Obuchowska in 1995 proposed another iterative procedure that checks, repeatedly, the existence of the implicit equality constraints. Theoretical characterization about the boundedness of (c-QP), however, does not have a complete result so far, except for Eaves' theorem, first by Eaves and later by Dostál, which answered the boundedness question only partially for a polyhedraltype of constraints. In this paper, Eaves' theorem is generalized to answer, necessarily and sufficiently, when the general (c-QP) with a convex quadratic constraint (not just a polyhedron) can be bounded from below, with a new insight that it can only be unbounded within an affine subspace.
\end{abstract}

\section{Introduction}

We are interested in looking for a necessary and sufficient condition to characterize the boundedness of the following convex quadratic programming problem (c-QP):

$$
\begin{array}{ll}
\min & f_{0}(x)=x^{T} Q_{0} x+2\left(b^{0}\right)^{T} x+\beta_{0} \\
\text { s.t. } & f_{i}(x)=x^{T} Q_{i} x+2\left(b^{i}\right)^{T} x+\beta_{i} \leq 0, \quad i=1,2, \ldots, m,
\end{array}
$$

where $Q_{i} \succeq 0 \in \mathbb{R}^{n \times n} ; b^{i} \in \mathbb{R}^{n} ; \beta_{i} \in \mathbb{R}$ for all $i=0,1,2, \ldots, m$. Let $I=\{1,2, \ldots, m\}$ and let the convex feasible set of 1.1 be denoted by

$$
C_{I}=\left\{x \in \mathbb{R}^{n} \mid f_{i}(x)=x^{T} Q_{i} x+2\left(b^{i}\right)^{T} x+\beta_{i} \leq 0, \forall i \in I\right\} .
$$

Notice that, in 1.2 , the notation $C_{I}$ emphasizes the index set $I$. For convenience, we define $I_{0}=I \cup\{0\}$ to include the objective function and

$$
C_{I_{0}}=\left\{x \in \mathbb{R}^{n} \mid f_{i}(x)=x^{T} Q_{i} x+2\left(b^{i}\right)^{T} x+\beta_{i} \leq 0, \forall i \in I_{0}\right\} .
$$

Received July 24, 2019; Accepted May 3, 2020.

Communicated by Jein-Shan Chen.

2010 Mathematics Subject Classification. 90C20, 90C22, 90C32.

Key words and phrases. convex quadratic programming, $l_{p}$ programming, Eaves theorem, recession cone. This work was supported by Taiwan Ministry of Science and Technology under grants MOST 105-2115M-006-005-MY2; MOST 106-2811-M-006 -021 and MOST 107-2811-M-006-009.

${ }^{*}$ Corresponding author. 
Similarly, if $I^{\prime} \subset I$, we can define $I_{0}^{\prime}=I^{\prime} \cup\{0\}$. Moreover, let

$$
C_{I^{\prime}}=\left\{x \in \mathbb{R}^{n} \mid f_{i}(x) \leq 0, \forall i \in I^{\prime}\right\} \quad \text { and } \quad C_{I_{0}^{\prime}}=\left\{x \in \mathbb{R}^{n} \mid f_{i}(x) \leq 0, \forall i \in I_{0}^{\prime}\right\} .
$$

Those notations will facilitate the explanations to our results later.

When $C_{I}$ is a polyhedron, i.e., $Q_{i}=0, \forall i \in I$, it was known first by Eaves in [5] and later by Dostál in [4] (see Corollary 3.2) that (1.1) is bounded from below if and only if

$$
\left(\left(b^{i}\right)^{T} v \leq 0, \forall i \in I\right) \wedge\left(Q_{0} v=0\right) \quad \Longrightarrow \quad\left(b^{0}\right)^{T} v \geq 0 .
$$

For the general convex set $C_{I}$, however, there is no counterpart yet, except for Kim et al. 6] who proposed a necessary but not sufficient; a sufficient but not necessary condition. See Corollary 3.3. Our main theorem below provides Condition (1.4) below to generalize (1.3) and answers the boundedness of 1.1 completely.

Theorem 1.1 (Main result). If $C_{I}$ is nonempty, then (1.1) is bounded from below if and only if there exists a subset $\emptyset \neq I^{\prime} \subset I$ such that all directions of recession in $0^{+} C_{I_{0}^{\prime}}$ are perpendicular to the subspace spanned by vectors $b_{i}, i \in I_{0}^{\prime}:=I^{\prime} \cup\{0\}$. That is, (1.1) is bounded from below if and only if there exists $\emptyset \neq I^{\prime} \subset I$ such that

$$
v \in 0^{+} C_{I_{0}^{\prime}} \quad \Longrightarrow \quad\left(b^{i}\right)^{T} v=0, \quad \forall i \in I_{0}^{\prime} .
$$

In literature, the boundedness of (1.1) used to be handled algorithmically. By doing the Cholesky factorization for each $Q_{i}, i \in I_{0}, 11.1$ can be cast into an $l_{p}$ programming problem (with $p=2$ ). The $l_{p}$ programming was first developed by Peterson and Ecker 9 11] and later by Terlaky 13 with a simpler proof. The dual of an $l_{p}$ programming problem has a complicated objective function but its constraint set is a polyhedron. Since it was proved in 911,13 that an $l_{p}$ programming problem is bounded from below if and only if its dual has a non-empty feasible domain, the boundedness of (1.1) can be thus determined by linear programming for testing the feasibility of the $l_{p}$ dual programming. In spite that the problem can be so resolved, we see almost no mathematical insight by this approach because the information was largely hidden behind the transformation into the dual of an $l_{p}$ programming.

Then, Caron and Obuchowska [2] in 1995 provided another iterative procedure for determining the boundedness of (1.1). Apparently, they did not know the above $l_{p}$ programming approach. In each iteration of Caron and Obuchowska's Algorithm A, they identify "implicit equality constraints" in a system of linear (in)equalities by linear programming. They showed that Algorithm A in [2] would either stop in Step 2 if (1.1) is unbounded; or in Step 3 if 1.1 is bounded. If one views the $l_{p}$ programming as a dual approach for determining the unboundedness of (1.1), Caron and Obuchowska's Algorithm A would be a nice primal algorithm. 
Although Caron and Obuchowska [2, Section 4] claimed that they have found the necessary and sufficient condition in the form of an algorithm for the boundedness of (1.1), yet a "mathematical" necessary and sufficient condition like Eaves' in (1.3) remains unknown until we prove Theorem 1.1 in this paper now. With Theorem 1.1, it becomes clear that Algorithm A simply checks the Condition (1.4) repeatedly and tries to find the largest index set $I^{\prime} \subset I$ satisfying (1.4). Caron and Obuchowska's algorithm is thus a nice implementation of Theorem 1.1 and it surprisingly came much earlier than our work.

The major difference in testing the unboundedness over a polyhedron $C_{I}\left(Q_{i}=0\right.$, $\forall i \in I$ ) and over a general $C_{I}$ lies in the fact that the former can be done by solely checking all recession directions of $C_{I}$ whereas the latter cannot. A typical example in [1,8] is provided here for better illustration.

$$
\min f_{0}(x)=-x_{1} \text { s.t. } f_{1}(x)=x_{1}^{2}-x_{2} \leq 0, \quad f_{2}(x)=-x_{1} \leq 0, \quad f_{3}(x)=-x_{2} \leq 0 .
$$

This example is unbounded from below, but not along any feasible ray. In fact, the only feasible ray of 1.5 is the positive $x_{2}$-axis

$$
v \in 0^{+} C_{I}=\{(0, t): t \geq 0\}
$$

along which the objective value

$$
\begin{aligned}
f_{0}\left(x_{0}+\alpha v\right) & =f_{0}\left(x_{0}\right)+\alpha^{2}\left(v^{T} Q_{0} v\right)+2 \alpha\left(v^{T} Q_{0} x_{0}+\left(b^{0}\right)^{T} v\right) \\
& =f_{0}\left(x_{0}\right), \quad \forall x_{0} \in C_{I}, \forall \alpha \geq 0
\end{aligned}
$$

stays unchanged regardless $x_{0} \in C_{I}$. Actually, problem 1.5 is unbounded from below along the parabola $f_{1}(x)=x_{1}^{2}-x_{2}=0$, not along the direction of recession of $C_{I}$.

As a consequence of this example, when $C_{I}$ is not a polyhedron, checking the unboundedness of the objective function $f_{0}(x)$ only for all recession directions of $C_{I}$ is just a very weak necessary condition. By Theorem 1.1 and Condition (1.4), the necessary and sufficient condition for (1.1) to be unbounded is that, for all possible subsets $I^{\prime} \in I$, at least one of $f_{i}(x), i \in I^{\prime} \cup\{0\}$ (not necessarily the objective function $f_{0}(x)$ itself) is unbounded from below along some common direction of recession in $0^{+} C_{I_{0}}$. The situation is way more complicated than that on a polyhedron $C_{I}$ so that our result is highly non-trivial.

Our technical skill to penetrate deeper into the structure is to write the objective function $f_{0}$, through a suitable coordinate transformation, as a canonical form having a sum-of-squares and one pure-linear term as follows (see (3.1)):

$$
f_{0}(x)=\lambda_{1} x_{1}^{2}+\lambda_{2} x_{2}^{2}+\cdots+\lambda_{p} x_{p}^{2}+\mu x_{p+1}, \quad \lambda_{i}>0, i=1, \ldots, p ; \mu>0
$$

where $p+1 \leq n$. With this canonical form, Theorem 3.1 (another version of Theorem 1.1 in terms of the canonical form) asserts that $f_{0}(x)$ is (un)bounded from below on $C_{I}$ if 
and only if $x_{p+1}$ is (un)bounded below on $C_{I}$ intersecting an affine subspace $\Theta\left(x^{0}\right)$, which involves only variables $x_{p+1}, x_{p+2}, \ldots, x_{n}$ :

$$
\Theta\left(x^{0}\right)=\left\{x \in \mathbb{R}^{n} \mid x=x^{0}+x_{p+1} e_{p+1}+\cdots+x_{n} e_{n}\right\}
$$

where $x^{0} \in C_{I}$ and $e_{i}$ is the $i$ th unit vector. It then reduces the boundedness of (1.1) to just checking the boundedness of a linear objective function $x_{p+1}$ on $C \cap \Theta\left(x^{0}\right)$.

The paper is organized as follows. In Section 2, we give examples to first understand the result of Theorem 1.1 and Condition (1.4). Section 3 is designated for the proof of Theorem 1.1 in the canonical form, which is Theorem 3.1. Then, in Corollary 3.2, we use Theorem 3.1 to prove Theorem 2.1 of [4] (Eaves theorem). In Corollary 3.3, we use Theorem 3.1 to prove also Theorem 2.1 of $[6]$ and finish the paper.

\section{Examples}

In this section, we use some examples to illustrate our main Theorem 1.1 and Condition (1.4).

Let $C$ be a convex set with its recession cone

$$
0^{+} C=\left\{v \in \mathbb{R}^{n}: x+t v \in C, \forall x \in C, \forall t \geq 0\right\}
$$

In particular, when $C=C_{I}$ is described by quadratic inequalities, its recession cone can be characterized by solving linear (in)equalities as follows [6, 12]:

$$
0^{+} C_{I}=\left\{v \in \mathbb{R}^{n}: Q_{i} v=0,\left(b^{i}\right)^{T} v \leq 0, \forall i \in I\right\}
$$

Furthermore, when $C_{I}$ is a polyhedron,

$$
0^{+} C_{I}=\left\{v \in \mathbb{R}^{n}:\left(b^{i}\right)^{T} v \leq 0, \forall i \in I\right\}
$$

\section{Example 2.1.}

$$
\min f_{0}(x)=-x_{1} \text { s.t. } f_{1}(x)=x_{1}^{2}-x_{2} \leq 0, \quad f_{2}(x)=-x_{1} \leq 0, \quad f_{3}(x)=-x_{2} \leq 0 \text {. }
$$

First, by $f_{0}=f_{2}$, we know that $0^{+} C_{I_{0}}=0^{+} C_{I}=\left\{\left(0, v_{2}\right): v_{2} \geq 0\right\}$. Moreover, since $b^{0}=b^{2}=(-1 / 2,0), b^{1}=b^{3}=(0,-1 / 2)$, it is easy to see that Condition (1.4) fails for any subset $I^{\prime} \subset I$. Applying Theorem 1.1, we conclude that the example is unbounded from below.

On the other hand, we see that $x^{k}=\left(k, k^{2}\right) \in C_{I}$ for all $k>0$, and $\left\{f_{0}\left(x^{k}\right)\right\} \rightarrow-\infty$ as $k \rightarrow+\infty$. It verifies above conclusion. 


\section{Example 2.2.}

(2.1) $\min f_{0}(x)=x_{1}^{2}+x_{2}$ s.t. $f_{1}(x)=x_{1}^{2}-x_{1} x_{2}+2 x_{2}^{2}-x_{3} \leq 0, \quad f_{2}(x)=x_{1}^{2}-x_{2}-1 \leq 0$.

For this example, $0^{+} C_{I_{0}}=\left\{v=\left(0,0, v_{3}\right)^{T} \in \mathbb{R}^{3} \mid v_{3} \geq 0\right\}$ and (1.4) is violated for the index $i=1$. So we only consider $I^{\prime}=\{2\}$. Then, $0^{+} C_{I_{0}^{\prime}}=\left\{v=\left(0,0, v_{3}\right)^{T} \in \mathbb{R}^{3} \mid v_{3} \in \mathbb{R}\right\}$. It is clear that $b^{0}=(0,1 / 2,0), b^{2}=(0,-1 / 2,0)$ and, for $v \in 0^{+} C_{I_{0}^{\prime}}$, there are $\left(b^{i}\right)^{T} v=0$, $i=0,2$. By Theorem 1.1. we conclude that 2.1) is bounded from below.

On the other hand, since $x^{0}=(0,0,0) \in C_{I}$, by $(1.6), \Theta\left(x^{0}\right)=\left\{\left(0, x_{2}, x_{3}\right) \mid x_{2}, x_{3} \in\right.$ $\mathbb{R}\}$. We see that $C_{I} \cap \Theta\left(x^{0}\right)=\left\{\left(0, x_{2}, x_{3}\right) \mid 2 x_{2}^{2}-x_{3} \leq 0,-x_{2}-1 \leq 0\right\}$, therefore $x_{2}$ is bounded from below on $C_{I} \cap \Theta\left(x^{0}\right)$. By Theorem 3.1, 2.1) is bounded from below, it verifies above conclusion.

\section{Proof of Theorem 1.1}

We first notice that, if $f_{0}$ is bounded from below on $\mathbb{R}^{n}$, it must be bounded below on $C_{I}$. To avoid triviality, we have the following assumptions.

A1. there is at least one $i \in I$ such that $Q_{i} \neq 0$;

A2. the constraint set $C_{I}$ is unbounded, equivalently, $0^{+} C_{I} \neq\{0\}[12]$;

A3. $f_{0}$ is unbounded from below on $\mathbb{R}^{n}$.

Now because the perpendicularity of directions of recession in $0^{+} C_{I_{0}^{\prime}}$ to the subspace spanned by vectors $b_{i}, i \in I_{0}^{\prime}:=I^{\prime} \cup\{0\}$ and the boundedness of convex QCQP (1.1) are invariant with respect to an orthogonal linear transformation of the space, so we just need to prove our main theorem in the case $f_{0}$ has the canonical form.

We now show that $f_{0}(x)$ (unbounded from below on $\mathbb{R}^{n}$ ) can be always transformed into the canonical form

$$
f_{0}(x)=\lambda_{1} x_{1}^{2}+\lambda_{2} x_{2}^{2}+\cdots+\lambda_{p} x_{p}^{2}+\mu x_{p+1}
$$

where $p+1 \leq n, \mu>0, \lambda_{i}>0, i=1, \ldots, p$. If $Q_{0} \neq 0$, then there exists an orthogonal matrix $P$ such that

$$
P^{T} Q_{0} P=\operatorname{diag}\left(\lambda_{1}, \ldots, \lambda_{p}, 0, \ldots, 0\right)
$$

where $\lambda_{1}>0, \ldots, \lambda_{p}>0$. Let $y=P^{T} x$ and represent $f_{0}(y)$ in the new variable $y$ as

$$
f_{0}(y)=\lambda_{1} y_{1}^{2}+\cdots+\lambda_{p} y_{p}^{2}+2 c_{1} y_{1}+\cdots+2 c_{p} y_{p}+c_{p+1} y_{p+1}+\cdots+c_{n} y_{n}+\beta_{0}
$$

Now let $z_{i}=y_{i}+c_{i} / \lambda_{i}$ for $i=1, \ldots, p$, and $z_{i}=y_{i}$ for $i=p+1, \ldots, n$, we have

$$
f_{0}(z)=\lambda_{1} z_{1}^{2}+\cdots+\lambda_{p} z_{p}^{2}+c_{p+1} z_{p+1}+\cdots+c_{n} z_{n}+\alpha_{0}
$$


for some $\alpha_{0} \in \mathbb{R}$. Since $f_{0}(z)$ is assumed to be unbounded from below, there must be some $p+1 \leq i \leq n$ such that $c_{i} \neq 0$. Let $c=c_{p+1} e_{p+1}+\cdots+c_{n} e_{n}, c \neq 0$. Create a new orthogonal basis $\left\{\theta_{1}, \theta_{2}, \ldots, \theta_{n}\right\}$ of $\mathbb{R}^{n}$ as follows.

Let $\theta_{1}=e_{1}, \theta_{2}=e_{2}, \ldots, \theta_{p}=e_{p}, \theta_{p+1}=c /|c|$ and $\theta_{p+2}, \ldots, \theta_{n}$ are chosen such that $\left\{\theta_{1}, \theta_{2}, \ldots, \theta_{n}\right\}$ becomes an orthogonal basis. Then in this new basis, $f_{0}(z)$ has the form of (3.1) after we remove the constant term. If $Q_{0}=0$, we can similarly transform $f_{0}(x)$ to have only one linear term $f_{0}(x)=\mu x_{1}$.

Let us also call the canonical form of (1.1) below as

$$
\begin{array}{ll}
\min & f_{0}(x)=\lambda_{1} x_{1}^{2}+\lambda_{2} x_{2}^{2}+\cdots+\lambda_{p} x_{p}^{2}+2 \mu x_{p+1} \quad(\mu>0) \\
\text { s.t. } & f_{i}(x)=x^{T} Q_{i} x+2\left(b^{i}\right)^{T} x+\beta_{i} \leq 0, \quad j \in I .
\end{array}
$$

Then,

$$
Q_{0}=\operatorname{diag}\left(\lambda_{1}, \lambda_{2}, \ldots, \lambda_{p}, 0, \ldots, 0\right), \quad b^{0}=\mu e_{p+1}
$$

and $p=0$ if $Q_{0}=0$. We see that, if $f_{0}(x)$ is unbounded from below on $C_{I}$, then $x_{p+1}$ must be unbounded from below on $C_{I}$; but not conversely. For example, let $f_{0}(x)=x_{1}^{2}+x_{2}$ and $C_{I}=\left\{x \in \mathbb{R}^{2} \mid x_{1}^{2}-x_{2} \leq 0\right\}$. Then $x_{2}$ is unbounded in $C_{I}$ but $f_{0}(x)$ is bounded from below on $C_{I}$. So we need to find conditions for $x_{p+1}$ to be unbounded from below on $C_{I}$ in that case. For $x^{0} \in C_{I}$, let $\Theta\left(x^{0}\right)$ be the affine subspace of $n-p$ dimension passing through $x^{0}$ as defined in 1.6$)$. When $p=0, \Theta\left(x^{0}\right)=\mathbb{R}^{n}$ and $f_{0}(x)$ is simply reduced to $f_{0}(x)=2 \mu x_{1}$. We now arrive at our main result.

Theorem 3.1. Under Assumptions A1, A2, A3 and $f_{0}$ was transformed into the canonical form (3.1), the following three statements are equivalent.

(i) The function $f_{0}$ is bounded from below on $C_{I}$.

(ii) The variable $x_{p+1}$ is bounded from below on $C_{I} \cap \Theta\left(x^{0}\right)$.

(iii) There exists a subset $\emptyset \neq I^{\prime} \subset I$ such that

$$
v \in 0^{+} C_{I^{\prime} \cup\{0\}} \quad \Longrightarrow \quad\left(e_{p+1}\right)^{T} v=0 \wedge\left(\left(b^{i}\right)^{T} v=0, \forall i \in I^{\prime}\right) .
$$

Proof. The proof is constructed in the cycle (i) $\Rightarrow$ (ii) $\Rightarrow$ (iii) $\Rightarrow$ (i) by way of contradiction.

(i) $\Rightarrow$ (ii). Suppose that $f_{0}(x)$ is bounded from below on $C_{I}$ but $x_{p+1}$ is unbounded from below in $C_{I} \cap \Theta\left(x^{0}\right)$. Then there exists a sequence $\left\{x^{k}\right\} \subset C_{I} \cap \Theta\left(x^{0}\right)$ such that $x^{k}=x^{0}+t_{p+1}^{k} e_{p+1}+\cdots+t_{n}^{k} e_{n}$ and $t_{p+1}^{k} \rightarrow-\infty$ as $k \rightarrow \infty$. Therefore,

$$
f_{0}\left(x^{k}\right)=\lambda_{1}\left(x_{1}^{0}\right)^{2}+\lambda_{2}\left(x_{2}^{0}\right)^{2}+\cdots+\lambda_{p}\left(x_{p}^{0}\right)^{2}+\mu\left(x_{p+1}^{0}+t_{p+1}^{k}\right) \rightarrow-\infty \quad \text { as } k \rightarrow \infty,
$$

which is a contradiction. 
(ii) $\Rightarrow$ (iii). Suppose that $x_{p+1}$ is bounded from below on $C_{I} \cap \Theta\left(x^{0}\right)$ but $(3.3)$ is violated for all $\emptyset \neq I^{\prime} \subset I$. In the following we only focus on the case when $I^{\prime}=I=\{1,2, \ldots, m\}$. All other cases for different $I^{\prime} \subset I$ can be proved similarly.

(For $I^{\prime}=I$ so $C_{I^{\prime}}=C_{I}$ ) Suppose that $v^{*} \in 0^{+} C_{I \cup\{0\}}$ and $\left(e_{p+1}\right)^{T} v^{*}<0$. Then, $Q_{0} v^{*}=0$ and $x^{0}+t v^{*} \in C_{I}$ for all $t \geq 0$. By $(3.2)$, we have $v_{i}^{*}=0, \forall i=1,2, \ldots, p$. Thus, $x(t)=x^{0}+t v^{*} \in C \cap \Theta\left(x^{0}\right)$ for all $t \geq 0$. Then

$$
x(t)_{p+1}=x_{p+1}^{0}+t v_{p+1}^{*}=x_{p+1}^{0}+t\left(e_{p+1}\right)^{T} v^{*} \rightarrow-\infty \quad \text { as } t \rightarrow \infty,
$$

which is a contradiction to (ii). So, if $x_{p+1}$ is bounded from below on $C_{I} \cap \Theta\left(x^{0}\right)$, then for all $v^{*} \in 0^{+} C_{I \cup\{0\}}$, there must be $\left(e_{p+1}\right)^{T} v^{*}=0$ and thus the $(p+1)^{\text {th }}$ component of $v^{*}, v_{p+1}^{*}=0$.

Suppose $j \in I$ is such that $v^{*} \in 0^{+} C_{I \cup\{0\}}$ and $\left(b^{j}\right)^{T} v^{*}<0$. Since $x_{p+1}$ is bounded from below on $C_{I} \cap \Theta\left(x^{0}\right)$ and since, for convex optimization, the boundedness implies attainment (see $[7]$ ), there exists $x^{*} \in C_{I} \cap \Theta\left(x^{0}\right)$ such that

$$
x^{*} \in \underset{C_{I} \cap \Theta\left(x^{0}\right)}{\operatorname{argmin}}\left(x_{p+1}\right) .
$$

Recall that, under Assumption (ii), for $x^{0} \in C_{I}$, and $v^{*} \in 0^{+} C_{I \cup\{0\}}$, there is $x(t)=$ $x^{0}+t v^{*} \in C_{I} \cap \Theta\left(x^{0}\right), \forall t \geq 0$ and $v_{i}^{*}=0, i=1,2, \ldots, p, p+1$. We claim that, $\forall x \in C_{I \backslash\{j\}} \cap \Theta\left(x^{0}\right)$, its $(p+1)^{\text {th }}$ component $x_{p+1}$ is bounded from below by $x_{p+1}^{*}$. Specifically, we want to prove that

$$
x_{p+1} \geq\left(x^{*}+v^{*}\right)_{p+1}=x_{p+1}^{*}=\min _{C_{I} \cap \Theta\left(x^{0}\right)}\left(x_{p+1}\right) .
$$

Otherwise, assume that there exists $\bar{x} \in C_{I \backslash\{j\}} \cap \Theta\left(x^{0}\right)$ such that

$$
\bar{x}_{p+1}<\left(x^{*}+v^{*}\right)_{p+1}=x_{p+1}^{*} .
$$

Let $\left(\bar{x}, x^{*}+v^{*}\right)$ be the line segment which connects, but not includes, the two end points $\bar{x}$, $x^{*}+v^{*}$. Notice from (3.6) that $z_{p+1}<x_{p+1}^{*}$ for any $z \in\left(\bar{x}, x^{*}+v^{*}\right)$. Since $v^{*} \in 0^{+} C_{I \cup\{0\}}$, we know that $Q_{j} v^{*}=0$. Then,

$$
\begin{aligned}
f_{j}\left(x^{*}+v^{*}\right) & =\left(x^{*}+v^{*}\right)^{T} Q_{j}\left(x^{*}+v^{*}\right)+2\left(b^{j}\right)^{T}\left(x^{*}+v^{*}\right)+\beta_{j} \\
& =f_{j}\left(x^{*}\right)+2\left(b^{j}\right)^{T} v^{*} \\
& <f_{j}\left(x^{*}\right) \leq 0 .
\end{aligned}
$$

That is, $x^{*}+v^{*}$ is an interior point in $C_{j}=\left\{x \mid f_{j}(x) \leq 0\right\}$. By choosing $z \in\left(\bar{x}, x^{*}+v^{*}\right)$ sufficiently close to $x^{*}+v^{*}$, we have $f_{j}(z)<0$ and $z_{p+1}<x_{p+1}^{*}$. Since $C_{I} \subset C_{I \backslash\{j\}}$, we have $x^{*}+v^{*} \in C_{I} \cap \Theta\left(x^{0}\right) \subset C_{I \backslash\{j\}} \cap \Theta\left(x^{0}\right)$, so that

$$
\left(\bar{x}, x^{*}+v^{*}\right) \subset C_{I \backslash\{j\}} \cap \Theta\left(x^{0}\right) .
$$


Then, $z \in C_{I \backslash\{j\}} \cap \Theta\left(x^{0}\right)$, which, together with $f_{j}(z)<0$, implies that $z \in C_{I} \cap \Theta\left(x^{0}\right)$ and $z_{p+1}<x_{p+1}^{*}$. This contradicts (3.4). We have thus obtained (3.5). That is,

$$
\min _{C_{I \backslash\{j\}} \cap \Theta\left(x^{0}\right)}\left(x_{p+1}\right) \geq \min _{C \cap \Theta\left(x^{0}\right)}\left(x_{p+1}\right) .
$$

Conversely, since $C_{I} \cap \Theta\left(x^{0}\right) \subset C_{I \backslash\{j\}} \cap \Theta\left(x^{0}\right)$, there also is

$$
\min _{C_{I \backslash\{j\}} \cap \Theta\left(x^{0}\right)}\left(x_{p+1}\right) \leq \min _{C_{I} \cap \Theta\left(x^{0}\right)}\left(x_{p+1}\right) .
$$

As a result, under Assumption (ii), if (iii) is violated for the subset $I^{\prime}=I$, there always exists $j \in I$ such that

$$
\min _{C_{I \backslash\{j\}} \cap \Theta\left(x^{0}\right)}\left(x_{p+1}\right)=\min _{C_{I} \cap \Theta\left(x^{0}\right)}\left(x_{p+1}\right) .
$$

In fact, the same argument applies to all other subsets $I^{\prime} \subset I$. In particular, for $\emptyset \neq I^{\prime}=I \backslash\{j\}$, there exists again $k \in I \backslash\{j\}$ such that

$$
\min _{C_{I^{\prime} \backslash\{j, k\}} \cap \Theta\left(x^{0}\right)}\left(x_{p+1}\right)=\min _{C_{I \backslash\{j\}} \cap \Theta\left(x^{0}\right)}\left(x_{p+1}\right)=\min _{C_{I} \cap \Theta\left(x^{0}\right)}\left(x_{p+1}\right) .
$$

Continuing the process, we shall arrive at the following chain of equalities:

$$
\min _{C_{I} \cap \Theta\left(x^{0}\right)}\left(x_{p+1}\right)=\min _{C_{I \backslash\{j\}} \cap \Theta\left(x^{0}\right)}\left(x_{p+1}\right)=\min _{C_{I \backslash\{j, k\}} \cap \Theta\left(x^{0}\right)}\left(x_{p+1}\right)=\cdots=\min _{\Theta\left(x^{0}\right)}\left(x_{p+1}\right) .
$$

On the left-hand side, $\min _{C_{I} \cap \Theta\left(x^{0}\right)}\left(x_{p+1}\right)$ is bounded from below by Assumption (ii), while on the right-hand side, the infimum of $x_{p+1}$ over the affine space $\Theta\left(x^{0}\right)$ is negative infinity. The contradiction proves (ii) $\Rightarrow$ (iii).

(iii) $\Rightarrow$ (i). Let $\emptyset \neq I^{\prime} \subset I$ be the index set satisfying (3.3) but the function $f_{0}(x)$ is unbounded from below on $C_{I}$. Let $I_{0}^{\prime}=I^{\prime} \cup\{0\}$ and

$$
C_{I_{0}^{\prime}}=\left\{x \in \mathbb{R}^{n} \mid f_{0}(x) \leq 0, f_{i}(x) \leq 0, \forall i \in I^{\prime}\right\}
$$

Since $f_{0}(x)$ is unbounded below on $C_{I}$, it is unbounded below on $C_{I_{0}^{\prime}}$. Therefore, $C_{I_{0}^{\prime}}$ is an unbounded set and its recession cone $0^{+} C_{I_{0}^{\prime}} \supsetneqq\{0\}$. Moreover, due to Assumption (3.3),

$$
0^{+} C_{I_{0}^{\prime}}=\left\{v \in \mathbb{R}^{n} \mid Q_{i} v=0,\left(b^{i}\right)^{T} v=0, \forall i \in I_{0}^{\prime}\right\}
$$

is a vector space. Let $\left\{v_{1}, \ldots, v_{k}\right\}$ be an orthonormal basis of $0^{+} C_{I_{0}^{\prime}}$, and define $H_{a}^{v_{j}}$ to be the hyperplane passing through $a \in C_{I_{0}^{\prime}}$ with the normal vector $v_{j}$ :

$$
H_{a}^{v_{j}}=\left\{x \in \mathbb{R}^{n} \mid v_{j}^{T}(x-a)=0\right\}, \quad j=1,2, \ldots, k .
$$

Then, $C_{I_{0}^{\prime}} \cap H_{a}^{v_{j}} \neq \emptyset, \forall j=1,2, \ldots, k$, so we can define, for each $1 \leq \eta \leq k$,

$$
C_{I_{0}^{\prime}}^{\eta}(a):=C_{I_{0}^{\prime}} \cap\left(\bigcap_{j=1}^{\eta} H_{a}^{v_{j}}\right) .
$$


We first claim that

$$
\inf _{x \in C_{I_{0}^{\prime}}^{1}(a)} f_{0}(x)=\inf _{x \in C_{I_{0}^{\prime}}^{1}(b)} f_{0}(x), \quad \forall a, b \in C_{I_{0}^{\prime}} .
$$

Notice that $H_{b}^{v_{1}}$ is a translation of $H_{a}^{v_{1}}$ along $v_{1}$ so that $H_{a}^{v_{1}}+\lambda v_{1}=H_{b}^{v_{1}}$. We can assume $\lambda \geq 0$, otherwise just exchange the role of $a$ and $b$. Now, we have

$$
C_{I_{0}^{\prime}}^{1}(a)+\lambda v_{1}=C_{I_{0}^{\prime}}^{1}(b) .
$$

Indeed, if $x+\lambda v_{1} \in C_{I_{0}^{\prime}}^{1}(a)+\lambda v_{1}$ then $x \in C_{I_{0}^{\prime}}^{1}(a)$ so $x \in C_{I_{0}^{\prime}}$ and $x \in H_{a}^{v_{1}}$. Since $v_{1} \in 0^{+} C_{I_{0}^{\prime}}$ and $\lambda \geq 0$, we have $x+\lambda v_{1} \in C_{I_{0}^{\prime}}$. By translation, we have $x+\lambda v_{1} \in H_{b}^{v_{1}}$. So $x+\lambda v_{1} \in C_{I_{0}^{\prime}} \cap H_{b}^{v_{1}}=C_{I_{0}^{\prime}}^{1}(b)$. Conversely, if $y \in C_{I_{0}^{\prime}}^{1}(b)$ then $y \in C_{I_{0}^{\prime}}$ and $y \in H_{b}^{v_{1}}$. Then there is $x \in H_{a}^{v_{1}}$ such that $y=x+\lambda v_{1}$. Note that $Q_{i} v_{1}=0$ and $\left(b^{i}\right)^{T} v_{1}=0$ for all $i \in I_{0}^{\prime}$ so $f_{i}(x)=f_{i}(y)$ and this implies that $x \in C_{I_{0}^{\prime}}$. So $x \in C_{I_{0}^{\prime}} \cap H_{a}^{v_{1}}=C_{I_{0}^{\prime}}^{1}(a)$ and therefore $y \in C_{I_{0}^{\prime}}^{1}(a)+\lambda v_{1}$. The equation 3.7 is proved. Then we have

$$
\inf _{x \in\left(C_{I_{0}^{\prime}}^{1}(a)+\lambda v_{1}\right)} f_{0}(x)=\inf _{x \in C_{I_{0}^{\prime}}^{\prime}(b)} f_{0}(x) .
$$

But $f_{0}\left(x+\lambda v_{1}\right)=f_{0}(x)$ for all $x \in C_{I_{0}^{\prime}}^{1}(a)$, so it holds that

$$
\inf _{x \in C_{I_{0}^{\prime}}^{1}(a)} f_{0}(x)=\inf _{x \in C_{I_{0}^{\prime}}^{1^{\prime}}(b)} f_{0}(x), \quad \forall a, b \in C_{I_{0}^{\prime}} .
$$

This shows that

$$
\inf _{C_{I_{0}^{\prime}}^{1}(a)} f_{0}(x)=\inf _{\bigcup_{b \in C_{I_{0}^{\prime}}} C_{I_{0}^{\prime}}^{1}(b)} f_{0}(x)
$$

Now we note that $C_{I_{0}^{\prime}}=\bigcup_{b \in C_{I_{0}^{\prime}}}\{b\} \subset \bigcup_{b \in C_{I_{0}^{\prime}}} H_{b}^{v_{1}}$ so

$$
\bigcup_{b \in C_{I_{0}^{\prime}}} C_{I_{0}^{\prime}}^{1}(b)=\bigcup_{b \in C_{I_{0}^{\prime}}}\left(C_{I_{0}^{\prime}} \cap H_{b}^{v_{1}}\right)=C_{I_{0}^{\prime}} \cap\left(\bigcup_{b \in C_{I_{0}^{\prime}}} H_{b}^{v_{1}}\right)=C_{I_{0}^{\prime}} .
$$

Equation 3.8 can thus be written as

$$
\inf _{C_{I_{0}^{\prime}}^{1}(a)} f_{0}(x)=\inf _{\bigcup_{b \in C_{I_{0}^{\prime}}} C_{I_{0}^{\prime}}^{1}(b)} f_{0}(x)=\inf _{C_{I_{0}^{\prime}}} f_{0}(x) .
$$

Since $C_{I_{0}^{\prime}}^{2}(a)=C_{I_{0}^{\prime}} \cap\left(\bigcap_{\iota=1}^{2} H_{a}^{v_{\iota}}\right)=\left(C_{I_{0}^{\prime}} \cap H_{a}^{v_{1}}\right) \cap H_{a}^{v_{2}}=C_{I_{0}^{\prime}}^{1}(a) \cap H_{a}^{v_{2}}$ and using (3.9), we can replace $C_{I_{0}^{\prime}}^{1}(a)$ with $C_{I_{0}^{\prime}}$ and apply the same argument as above to have $\inf _{x \in C_{I_{0}^{\prime}}^{2}(a)} f_{0}(x)=$ $\inf _{x \in C_{I_{0}^{\prime}}} f_{0}(x)$. Continuing the process, we arrive at

$$
\inf _{x \in C_{I_{0}^{\prime}}} f_{0}(x)=\inf _{x \in C_{I_{0}^{\prime}}^{I^{\prime}}(a)} f_{0}(x)=\inf _{x \in C_{I_{0}^{\prime}}^{2}(a)} f_{0}(x)=\cdots=\inf _{x \in C_{I_{0}^{\prime}}^{k}(a)} f_{0}(x) .
$$


By assumption that $\inf _{x \in C} f_{0}(x)=-\infty$, we have $\inf _{x \in C_{I_{0}^{\prime}}} f_{0}(x)=-\infty$. From (3.10) we have $\inf _{x \in C_{I_{0}^{\prime}}^{k}(a)} f_{0}(x)=\inf _{x \in C_{I_{0}^{\prime}}} f_{0}(x)=-\infty$ and $C_{I_{0}^{\prime}}^{k}(a)$ must be an unbounded set. So there exists $0 \neq w \in 0^{+}\left(C_{I_{0}^{\prime}}^{k}(a)\right)$, see $[1$. On the other hand,

$$
0^{+}\left(C_{I_{0}^{\prime}}^{k}(a)\right)=0^{+}\left(C_{I_{0}^{\prime}} \cap H_{a}^{v_{1}} \cap \cdots \cap H_{a}^{v_{k}}\right)
$$

and $0^{+}\left(C_{I_{0}^{\prime}} \cap H_{a}^{v_{1}} \cap \cdots \cap H_{a}^{v_{k}}\right)=0^{+} C_{I_{0}^{\prime}} \cap 0^{+}\left(H_{a}^{v_{1}} \cap \cdots \cap H_{a}^{v_{k}}\right)$, see [12]. Then, $w \in 0^{+}\left(C_{I_{0}^{\prime}}\right)$ and $w \in 0^{+}\left(H_{a}^{v_{1}} \cap \cdots \cap H_{a}^{v_{k}}\right)=\bigcap_{i=1}^{k} 0^{+} H_{a}^{v_{i}}$. We note that $0^{+} C_{I_{0}^{\prime}}:=V$ is a vector space and, since each $H_{a}^{v_{i}}$ is an affine subspace, $0^{+} H_{a}^{v_{i}}$ is a vector subspace which is a translation of $H_{a}^{v_{i}}$. So we have

$$
0^{+}\left(H_{a}^{v_{1}} \cap \cdots \cap H_{a}^{v_{k}}\right)=\bigcap_{i=1}^{k} 0^{+} H_{a}^{v_{i}}=V^{\perp} .
$$

This implies that $0 \neq w \in V \cap V^{\perp}$. This contradiction indicates that $f_{0}(x)$ must be bounded on $C_{I}$ under the hypothesis (iii).

To end this section, we will show that main results in [4] and [6] are corollaries of Theorem 1.1 .

Corollary 3.2. [4, Theorem 2.1] If $C_{I}$ is nonempty and $Q_{i}=0$ for all $i \in I$, then (1.1) has a solution if and only if the following condition is satisfied:

$$
\left(\left(b^{i}\right)^{T} v \leq 0, \forall i \in I, Q_{0} v=0\right) \quad \Longrightarrow \quad\left(b^{0}\right)^{T} v \geq 0 .
$$

Proof. $(\Rightarrow)$ When (1.1) has a solution, it means that (1.1) is bounded from below on $C_{I}$. Please see [7]. We need to prove that (3.11) occurs. Suppose on the contrary that there exists $v^{*}$ such that

$$
\left(b^{i}\right)^{T} v^{*} \leq 0, \quad \forall i \in I, \quad Q_{0} v^{*}=0 \text { but }\left(b^{0}\right)^{T} v^{*}<0 .
$$

Then 3.12 implies that 1.4$)$ is violated for any $I^{\prime} \subset I$. By the negation of Theorem 1.1 , (1.1) must be unbounded from below, which is a contradiction.

$(\Leftarrow)$ Assume that 3.11 holds. We need to prove that 1.1 has a solution. To avoid triviality, we assume that $f_{0}$ is unbounded from below on $\mathbb{R}^{n}$ and $f_{0}$ adopts the canonical form: $f_{0}(x)=\lambda_{1} x_{1}^{2}+\lambda_{2} x_{2}^{2}+\cdots+\lambda_{p} x_{p}^{2}+2 \mu x_{p+1}(\mu>0)$. Then, $b^{0}=2 \mu e_{p+1}$. Moreover, from (3.11), we immediately have

$$
\left(v^{T} Q_{0}=0, v^{T} b^{i} \leq 0, \forall i \in I_{0}=I \cup\{0\}\right) \quad \Longrightarrow \quad v^{T} b^{0}=0 .
$$

Let $x^{0} \in C_{I}$ and define

$$
\bar{C}=C_{I} \cap \Theta\left(x^{0}\right) \cap\left\{x: x_{p+1} \leq x_{p+1}^{0}\right\} .
$$


Since $C_{I}$ is a polyhedron, $\bar{C}$ is, too. By Minkowski-Weyl theorem, there exists $c^{1}, \ldots, c^{j} \in$ $\mathbb{R}^{n}$ such that

$$
\bar{C}=0^{+}(\bar{C})+\operatorname{conv}\left(c^{1}, \ldots, c^{j}\right),
$$

where conv denotes the convex hull. Since $\Theta\left(x^{0}\right)$ involves only variables $x_{p+1}, x_{p+2}, \ldots, x_{n}$, to keep $x+t v \in \Theta\left(x^{0}\right) \cap\left\{x: x_{p+1} \leq x_{p+1}^{0}\right\}, x \in \Theta\left(x^{0}\right) \cap\left\{x: x_{p+1} \leq x_{p+1}^{0}\right\}, t \geq 0$, it is necessary that $v_{1}=v_{2}=\cdots=v_{p}=0$ and $v_{p+1} \leq 0$. In other words, we have $v^{T} Q_{0}=0$ and $v^{T} b^{0}=2 \mu v_{p+1} \leq 0$ so that

$$
0^{+}(\bar{C})=\left\{v: v^{T} Q_{0}=0, v^{T} b^{i} \leq 0, \forall i \in I_{0}\right\} .
$$

Then, 3.13 implies that, for $v \in 0^{+}(\bar{C})$, there is $v_{p+1}=0$. On the other hand, the convex hull conv $\left(c^{1}, \ldots, c^{j}\right)$ is finitely generated and therefore bounded. As a result, the $(p+1)^{\text {th }}$ component of vectors in $\bar{C}$ is bounded. Therefore $x_{p+1}$ is bounded from below on $C_{I} \cap \Theta\left(x^{0}\right)$. Applying Theorem 3.1, we have $f_{0}$ is bounded from below on $C_{I}$, so (1.1) has a solution.

Corollary 3.3. 6, Theorem 2.1] Let $C_{I} \neq \emptyset$.

(i) If (1.1) has a solution, then

$$
\left(v \in 0^{+} C_{I}, Q_{0} v=0\right) \quad \Longrightarrow \quad\left(b^{0}\right)^{T} v \geq 0 .
$$

(ii) If either (3.14) holds and $b^{0}=0$ or the condition

$$
\left(v \in 0^{+} C_{I} \backslash\{0\}, Q_{0} v=0\right) \quad \Longrightarrow \quad\left(b^{0}\right)^{T} v>0
$$

is satisfied, then (1.1) has a solution.

Proof. The proof of (i) is analogous to the necessary part of the proof for Corollary 3.2.

For (ii), using the canonical form of $f_{0}$, it is clear that $b^{0}=0$ implies that $f_{0}$ is bounded from below on $\mathbb{R}^{n}$ and thus on $C_{I}$. When $b^{0} \neq 0$ but 3.15 holds, this immediately implies that $0^{+}\left(C_{I_{0}}\right) \backslash\{0\}=\emptyset$. That is, $0^{+}\left(C_{I_{0}}\right)=\{0\}$. Then, $\left(\forall i \in I_{0}\right) Q_{i} v=0,\left(b^{i}\right)^{T} v \leq 0 \Rightarrow$ $\left(b^{i}\right)^{T} v=0$. By Theorem 1.1, $f_{0}$ is bounded from below on $C_{I}$ and thus (1.1) has a solution.

\section{Concluding remarks}

In this paper, we generalize Eaves' theorem [4 6] to give a theoretical characterization for the boundedness of problem (1.1) and show clearly that if 1.1 is unbounded from below, the unboundedness happens only on a translation of the null space of $Q_{0}$. On the other hand, our result provides another angle to see how the function $f_{0}(x)$ is unbounded on $C_{I}$ if it is (see statement (ii) of Theorem 3.1) and also a complete answer to the question proposed by Kim et al. in [6]. Finally, we believe that our technique can be extended to complete the results obtained by Dong and Tam in their paper [3]. 


\section{References}

[1] R. J. Caron and W. Obuchowska, Unboundedness of a convex quadratic function subject to concave and convex quadratic constraints, European J. Oper. Res. 63 (1992), no. $1,114-123$.

$[2] \_$, An algorithm to determine boundedness of quadratically constrained convex quadratic programmes, European J. Oper. Res. 80 (1995), no. 2, 431-438.

[3] V. V. Dong and N. N. Tam, On the solution existence of convex quadratic programming problems in Hilbert spaces, Taiwanese J. Math. 20 (2016), no. 6, 1417-1436.

[4] Z. Dostál, On solvability of convex noncoercive quadratic programming problems, J. Optim. Theory Appl. 143 (2009), no. 2, 413-416.

[5] B. C. Eaves, On quadratic programming, Management Sci. 17 (1971), 698-711.

[6] D. S. Kim, N. N. Tam and N. D. Yen, Solution existence and stability of quadratically constrained convex quadratic programs, Optim. Lett. 6 (2012), no. 2, 363-373.

[7] Z.-Q. Luo and S. Zhang, On extensions of the Frank-Wolfe theorems, Comput. Optim. Appl. 13 (1999), no. 1-3, 87-110.

[8] K. G. Murty, Linear Complementarity, Linear and Nonlinear Programming, Sigma Series in Applied Mathematics 3, Heldermann Verlag, Berlin, 1988.

[9] E. L. Peterson and J. G. Ecker, Geometric programming: duality in quadratic programming and $l_{p}$-approximation I, Proceedings of the Princeton Symposium on Mathematical Programming (Princeton Univ., 1967), 445-480, Princeton University Press, Princeton, N.J., 1970.

[10] _ Geometric programming: duality in quadratic programming and $l_{p^{-}}$ approximation II (Canonical programs), SIAM J. Appl. Math. 17 (1969), 317-340.

[11] _ Geometric programming: duality in quadratic programming and $l_{p^{-}}$ approximation III: Degenerate programs, J. Math. Anal. Appl. 29 (1970), 365-383.

[12] R. T. Rockafellar, Convex Analysis, Princeton Mathematical Series 28, Princeton University Press, Princeton, N.J., 1970.

[13] T. Terlaky, On $l_{p}$ programming, European J. Oper. Res. 22 (1985), no. 1, 70-100. 
Huu-Quang Nguyen

Department of Mathematics, School of Natural Sciences Education, Vinh University, Vinh, Nghe An, Vietnam

and

Department of Mathematics, National Cheng Kung University, Taiwan

E-mail address: quangdhv@gmail.com

Van-Bong Nguyen

Department of Mathematics, Tay Nguyen University, Vietnam

E-mail address: nvbong@ttn.edu.vn

Ruey-Lin Sheu

Department of Mathematics, National Cheng Kung University, Taiwan

E-mail address: rsheu@mail.ncku.edu.tw 